\title{
Brain white matter structure and information processing speed in healthy older age
}

\author{
Ksenia A. Kuznetsova ${ }^{1,2} \cdot$ Susana Muñoz Maniega ${ }^{3,4,5}$ - Stuart J. Ritchie ${ }^{5,6}$. \\ Simon R. Cox ${ }^{5,6}$ • Amos J. Storkey ${ }^{2} \cdot$ John M. Starr $^{5,7} \cdot$ Joanna M. Wardlaw $^{3,4,5}$. \\ Ian J. Deary ${ }^{5,6} \cdot$ Mark E. Bastin ${ }^{3,4,5}$
}

Received: 14 May 2015/Accepted: 29 July 2015/Published online: 9 August 2015

(C) The Author(s) 2015. This article is published with open access at Springerlink.com

\begin{abstract}
Cognitive decline, especially the slowing of information processing speed, is associated with normal ageing. This decline may be due to brain cortico-cortical disconnection caused by age-related white matter deterioration. We present results from a large, narrow age range cohort of generally healthy, community-dwelling subjects in their seventies who also had their cognitive ability tested in youth (age 11 years). We investigate associations between older age brain white matter structure, several measures of information processing speed and childhood cognitive ability in 581 subjects. Analysis of diffusion tensor MRI data using Tract-based Spatial Statistics
\end{abstract}

I. J. Deary, M. E. Bastin contributed equally to the manuscript.

Ian J. Deary

I.Deary@ed.ac.uk

$\square$ Mark E. Bastin

Mark.Bastin@ed.ac.uk

1 Doctoral Training Centre in Neuroinformatics and Computational Neuroscience, School of Informatics, University of Edinburgh, Edinburgh, UK

2 Institute for Adaptive and Neural Computation, School of Informatics, University of Edinburgh, Edinburgh, UK

3 Brain Research Imaging Centre, Neuroimaging Sciences, University of Edinburgh, Edinburgh, UK

4 Scottish Imaging Network, A Platform for Scientific Excellence (SINAPSE) Collaboration, Edinburgh, UK

5 Centre for Cognitive Ageing and Cognitive Epidemiology (CCACE), University of Edinburgh, 7 George Square, Edinburgh EH8 9JZ, UK

6 Department of Psychology, University of Edinburgh, 7 George Square, Edinburgh EH8 9JZ, UK

7 Alzheimer Scotland Dementia Research Centre, University of Edinburgh, Edinburgh, UK
(TBSS) showed that all measures of information processing speed, as well as a general speed factor composed from these tests $\left(g_{\text {speed }}\right)$, were significantly associated with fractional anisotropy (FA) across the white matter skeleton rather than in specific tracts. Cognitive ability measured at age 11 years was not associated with older age white matter FA, except for the $g_{\text {speed }}$-independent components of several individual processing speed tests. These results indicate that quicker and more efficient information processing requires global connectivity in older age, and that associations between white matter FA and information processing speed (both individual test scores and $g_{\text {speed }}$ ), unlike some other aspects of later life brain structure, are generally not accounted for by cognitive ability measured in youth.

Keywords Ageing - White matter structure - Information processing speed · Diffusion MRI

\section{Introduction}

Cognitive ageing is associated with decline in average levels of some cognitive capabilities, such as information processing speed, abstract reasoning, executive function and aspects of memory, even in the absence of overt pathology (Salthouse 2010). Understanding the biological bases of cognitive ageing is therefore an increasingly important area of research for developed societies with ageing populations (Deary et al. 2007, 2009). Brain structural changes are also commonly observed in normal ageing and include atrophy of both grey and white matter regions (Bartzokis et al. 2001; Raz et al. 2005), ventricular enlargement, white matter hyperintensities (WMH) in deep and periventricular regions (Fazekas et al. 1987), enlarged 
perivascular spaces (Doubal et al. 2010) and mineral deposition (Bartzokis et al. 2007). The present study focuses on cerebral white matter, and hence the brain's ability to pass information efficiently between different cortical regions, an important factor in terms of understanding cognitive functioning in older age (GunningDixon et al. 2009; Malloy et al. 2007; Penke et al. 2012).

It has been argued that information processing speed is a fundamental characteristic of the brain's cognitive efficiency. This is because it is relatively elementary; the tests which assess it require only simple and repetitive responses to questions which would not attract errors if given sufficient time. Performance on simple tests of processing speed is associated with scores on more complex cognitive tests, such as reasoning (Jensen 2006). Processing speed might therefore mediate how age-related brain changes influence the efficiency of 'higher' cognitive functions (Deary 2000; Salthouse 1996; Verhaeghen 2014; Penke et al. 2012).

A number of cognitive tests with different stimulusresponse arrangements can provide measures of processing speed. These include paper-and-pencil psychometric tests, such as Digit-Symbol Substitution (Wechsler 1998), as well as psychophysical measurements, such as Choice Reaction Time (Deary et al. 2001; Jensen 1987) and Inspection Time (Deary 2000; Deary et al. 2010). Performance on processing speed tests is correlated; subjects who are more efficient on one speed test tend to be more efficient on the others. Therefore, data reduction techniques, which have the advantage of removing error and taskspecific variance associated with each individual test, can be used to combine an individual's performance on several tests into a general index of information processing speed (which we name ' $g_{\text {speed }}$ '). This is typically the first unrotated component (sometimes called a 'factor') from a principal component analysis (PCA) of scores from the individual tests which captures the largest proportion of the covariance between them (Luciano et al. 2009). Information processing speed, and hence $g_{\text {speed }}$, decreases with age and may therefore provide a useful indicator of cognitive decline in later life (Deary et al. 2010; Ritchie et al. 2014; Salthouse 1996; Verhaeghen 2014).

Brain white matter structure in older age can be investigated using imaging biomarkers derived from diffusion tensor MRI (DT-MRI), such as fractional anisotropy (FA) and mean diffusivity (MD) (Salat et al. 2005; Sullivan and Pfefferbaum 2006). Although ascribing these biomarkers directly to white matter 'integrity' is problematic (Jones et al. 2013), a number of authors have used them to investigate links between brain structure and processing speed in older age. For example, Kennedy and Raz (2009) examined relationships between FA measured in several white matter regions and cognitive tests, including processing speed, in a sample of 52 subjects aged between 19 and 81 years. After correcting for the effects of age, only working memory and processing speed remained significantly associated with FA in anterior brain regions. Haász et al. (2013) investigated links between general fluid intelligence $\left(g_{\mathrm{f}}\right)$ and white matter water diffusion parameters in a cohort of 100 healthy participants aged between 49 and 80 years. Using two measures of processing speed, a colour-word interference test and a visuo-spatial attention task, as well as several other tests to compute $g_{\mathrm{f}}$, they found associations between $g_{\mathrm{f}}$ and MD, FA and radial diffusivity across the white matter skeleton with Tract-based Spatial Statistics (TBSS; Smith et al. 2006). However, of all $g_{\mathrm{f}}$ tests, processing speed scores contributed most towards associations with white matter structure.

Vernooij et al. (2009) investigated relationships between brain white matter water diffusion parameters and cognitive ability in 860 people aged 60 and older. They found that elevated mean, axial and radial diffusivities and decreased FA were associated with worse performance on processing speed and general cognitive ability tasks for both normal-appearing white matter and that located within WMH. In their study of 554 adults aged 70-82 years, van den Heuvel et al. (2006) found that periventricular WMH were significantly associated with decreased processing speed performance as measured by the Letter-Digit Coding test.

Although these studies provide valuable insights into links between brain white matter structure and cognition in older age, they tend to include participants with a wide range of ages. This is problematic since chronological age is a major confounder of cognition and white matter structure, and age-heterogeneous cohorts may mask its true effect (Hofer and Sliwinski 2001); for example, white matter FA and MD are known to decrease and increase, respectively, with advancing age requiring mediation analyses to account for such effects in brain-cognition studies of older adults (Madden et al. 2012). In the present study, we investigate relationships between white matter structure and information processing speed using voxelbased methods in the Lothian Birth Cohort 1936 (LBC1936), a large group of generally healthy subjects in their seventies with a narrow age range. These attributes, coupled with the availability of a validated measure of cognitive ability from childhood and a diverse range of processing speed tests performed in older age, make this cohort valuable for studying associations between brain structure and cognition in older age.

Previous studies conducted in the LBC1936 have assessed associations between general factors of information processing speed ( $\left.g_{\text {speed }}\right)$, general intelligence $(g)$ and brain white matter DT-MRI biomarkers measured in tracts segmented using quantitative tractography. Penke et al. (2010) reported associations between tract-average white 
matter FA, MD, axial and radial diffusivity, and $g_{\text {speed }}$ in a pilot sample of the LBC1936 $(n=132)$. They found that $g_{\text {speed }}$ had a significant association with tract-average FA and MD in all eight major tracts investigated (genu and splenium of corpus callosum, and bilateral cingulum cingulate gyri, uncinate and arcuate fasciculi). This suggests that $g_{\text {speed }}$ is likely to be a property related to global brain connectivity rather than to specific tracts. Penke et al. (2010) also demonstrated that, like $g$, a general brain white matter FA factor (explaining $40 \%$ of the individual differences across all eight tracts) could be extracted from the tractography data and this factor was associated with $g_{\text {speed }}$ $(r=-0.24, p=0.007)$. In a further analysis conducted when all LBC1936 participants' brain imaging data were available, Penke et al. (2012) studied the relationships between tract-average FA, longitudinal relaxation time and magnetisation transfer ratio with $g$ and $g_{\text {speed }}$ in 12 major white matter tracts in 420 subjects (the eight tracts measured in Penke et al. (2010) plus bilateral inferior longitudinal fasciculi and anterior thalamic radiations). General factors of all three tract-averaged biomarkers (explaining $40-70 \%$ of the individual differences across all 12 tracts) were independently associated with $g$ (together explaining $10 \%$ of the variance) and, importantly, this effect was completely mediated by $g_{\text {speed }}$. This is amongst the strongest evidence to date that processing speed is a mediator between brain white matter structure and general cognitive functioning. However, these previous studies have measured imaging biomarkers averaged across specific tracts, and have not investigated whether, and where, individual tests might be correlated with white matter structure using methods that allow greater spatial localisation of effects.

The present paper reports new analyses on the LBC1936, testing the extent to which white matter structure is related to differences in processing speed measures. For these analyses, we used TBSS rather than quantitative tractography methods. TBSS allows analysis of DT-MRI data to be conducted on a voxel-wise basis across the brain, therefore revealing more detail on the spatial distribution of associations between white matter structure and cognitive ability than is possible with quantitative tractography. We first tested white matter FA associations with both $g_{\text {speed }}$ and the individual speed tests contributing to it. We also assessed whether specific aspects of the individual processing speed tests were associated with white matter structure beyond that accounted for by $g_{\text {speed }}$ (Booth et al. 2013). Finally, since our participants had measures of their childhood intelligence available, we tested whether later life associations between white matter FA and processing speed were still present after the speed tests had been adjusted for earlier cognitive ability.

\section{Methods}

\section{Participants}

The LBC1936 comprises 1091 community-dwelling individuals in their seventies who agreed to participate in a longitudinal study of cognitive ageing (Deary et al. 2007). At age 11 years, almost all of them took part in the Scottish Mental Survey of 1947, which employed the Moray House Test No. 12, a test of general cognitive ability. This test, which takes $45 \mathrm{~min}$ to complete, is mainly one of verbal reasoning, but includes a variety of other items on areas such as spatial abilities, arithmetic and cypher decoding (Scottish Council for Research in Education 1949). At recruitment in older age, subjects agreed to further cognitive testing and other medical, physical and psychosocial assessments at approximately 70 years of age (Deary et al. 2007). During the second wave of this study, and in addition to follow-up cognitive and health testing, participants also underwent a comprehensive MRI examination, including DT-MRI, to assess brain white matter structure at approximately 73 years of age (Wardlaw et al. 2011; Deary et al. 2012). Study inclusion criteria for the present report were that participants should have complete DT-MRI, childhood intelligence and age 73 processing speed data. Participants were also asked a series of Yes/No questions on their medical history by an interviewer. The questions determined whether participants had a history of cardiovascular disease or high cholesterol, and were being treated for hypertension, diabetes or blood circulation problems. Written informed consent was obtained from all subjects under protocols approved by the UK National Health Service Ethics Committees.

\section{Tests of information processing speed}

In the second wave of the study, and approximately contemporaneous with their brain MRI scan, subjects undertook a battery of cognitive tests designed to characterise a wide range of cognitive domains (Deary et al. 2007; Tucker-Drob et al. 2014). Processing speed was a particular focus of the study. Subjects undertook five processing speed tests, namely Digit-Symbol Substitution and Symbol Search from the Wechsler Adult Intelligence Scale, 3rd Edition (Wechsler 1998), Simple Reaction Time, FourChoice Reaction Time and Inspection Time; these tests are described in Deary et al. (2007).

For Digit-Symbol Substitution, participants were given a set of digit-symbol transformations as a 'dictionary' and asked to decode as many symbols as they could in two minutes. In Symbol Search, participants indicated whether or not one of a pair of symbols occurred in a row of 
symbols. The number of correct answers within the allotted time (two minutes) was recorded and used for subsequent analysis. Both tests were administered according to the standard instructions in the Wechsler manual.

Mean and SD (intra-individual variability) values for Simple and Four-Choice Reaction Time were measured using a portable reaction time device designed for the UK Health and Lifestyle Survey (Cox, Huppert and Whichelow 1993; Deary et al. 2001). The box includes a high-contrast LCD display and five response keys labelled 1, 2, 0, 3, 4 arranged in a shallow arc. For Simple Reaction Time, participants were asked to rest a finger on the 0 button; once a 0 appeared on the screen, the participants were told to press the button as rapidly as they could. The test consisted of 8 practice and 20 test trials. In the Four-Choice Reaction Time task, participants were told to place the second and middle fingers of each hand on the buttons labelled 1, 2, 3 and 4. Participants were then presented with a number (1, 2, 3 or 4) on the LCD screen, and were told to press the corresponding button as quickly as possible. The test consisted of a total of 48 trials: 8 practice and 40 test trials. Within the test trials, each of the numbers appeared 10 times in a random order. The time between trials varied randomly from 1 to $3 \mathrm{~s}$.

For Inspection Time, participants were exposed to a visual stimulus consisting of a pair of parallel vertical straight lines (connected with a horizontal bar at the top), one of which was markedly shorter than the other (Deary et al. 2004). The stimulus was displayed for varying durations, then immediately covered after offset by a visual pattern backward mask. The participants were asked to indicate the side (left or right) on which the longer line was present. The task does not involve the measurement of motor speed since there was no requirement for a fast response; the exposure time of the stimulus was varied and only the correctness of the response was used. The task consisted of 40 practice and 150 test trials with ten trials at each of 15 durations (6, $12,19,25,31,37,44,50,62,75,87,100,125,150$ and $200 \mathrm{~ms}$ ). The total number of correct responses was used as the measure of performance.

\section{General factor of information processing speed}

In order to capture the variance shared by these five processing speed tests, $g_{\text {speed }}$ was calculated using PCA. Only one component was indicated by scree slope analysis. Values of $g_{\text {speed }}$ were calculated for each participant as a projection of the five processing speed tests' loadings on the first unrotated principal component. In addition, residual, test-specific scores, namely the variance of each processing speed test not accounted for by $g_{\text {speed, }}$ were obtained from a confirmatory factor analysis of the five individual tests.

\section{Magnetic resonance imaging acquisition}

All MRI data were acquired using a GE Signa Horizon HDxt 1.5 T clinical scanner (General Electric, Milwaukee, WI, USA) using a self-shielding gradient set with maximum gradient strength of $33 \mathrm{mT} / \mathrm{m}$ and an 8 -channel phased-array head coil. The full details of the imaging protocol can be found in Wardlaw et al. (2011). Briefly, the DT-MRI examination consisted of $7 \quad \mathrm{~T}_{2}$-weighted $\left(b=0 \mathrm{~s} \mathrm{~mm}^{-2}\right)$ and sets of diffusion-weighted $\left(b=1000 \mathrm{~s} \mathrm{~mm}^{-2}\right) \quad$ single-shot spin-echo echo-planar (EP) volumes acquired with diffusion gradients applied in 64 non-collinear directions (Jones et al. 2002). Volumes were acquired in the axial plane, with a field-of-view of $256 \times 256 \mathrm{~mm}$, contiguous slice locations, and image matrix and slice thickness designed to give $2 \mathrm{~mm}$ isotropic voxels. The repetition and echo time for each EP volume were $16.5 \mathrm{~s}$ and $98 \mathrm{~ms}$, respectively.

\section{Image analysis}

All DT-MRI data were converted from DICOM (http:// dicom.nema.org) to NIfTI-1 (http://nifti.nimh.nih.gov/nifti1) format using the TractoR package for fibre tracking analysis (http://www.tractor-mri.org.uk). FSL tools (http:// www.fmrib.ox.ac.uk/fsl) were then used to extract the brain, remove bulk motion and eddy current-induced distortions by registering all subsequent volumes to the first $\mathrm{T}_{2}$-weighted EP volume (Jenkinson and Smith 2001), estimate the water diffusion tensor and calculate parametric maps of FA from its eigenvalues using DTIFIT.

\section{Tract-based spatial statistics}

Relationships between white matter FA, $g_{\text {speed }}$, individual test scores and their residuals were investigated across the brain using TBSS (http://www.fmrib.ox.ac.uk/fsl). First, all participants' FA volumes were linearly and non-linearly registered to a study-specific template. Second, a white matter skeleton was created from the mean of all registered FA volumes. This was achieved by searching for maximum FA values in directions perpendicular to the local tract direction in the mean FA volume. An FA threshold of 0.25 was applied to the skeleton to exclude predominantly nonwhite matter voxels. Third, for each subject's FA volume, the maximum voxel perpendicular to the local skeleton direction was projected onto the skeleton. This resulted in one FA skeleton volume per subject corresponding to centres of white matter structures. 
Table 1 Participant health characteristics

\begin{tabular}{|c|c|c|c|c|}
\hline & \multirow[t]{2}{*}{ Total sample } & \multicolumn{2}{|l|}{ Sex } & \multirow[t]{2}{*}{$p$ value } \\
\hline & & Male & Female & \\
\hline No. of subjects & 581 & 311 & 270 & \\
\hline MMSE & $28.85(1.25)$ & $28.73(1.31)$ & $28.98(1.16)$ & $>0.02$ \\
\hline Education (years) & $10.80(1.14)$ & $10.79(1.17)$ & $10.81(1.10)$ & 0.80 \\
\hline BMI $\left(\mathrm{kg} / \mathrm{m}^{2}\right)$ & $27.88(4.55)$ & $28.03(4.24)$ & $27.72(4.89)$ & 0.41 \\
\hline Alcohol consumption (units)* & $13.46(14.19)$ & $16.71(15.44)$ & $8.61(10.36)$ & $>0.001$ \\
\hline \multicolumn{5}{|l|}{ Smoking status (\%) } \\
\hline Never & $273(47.0)$ & $134(23.1)$ & $139(23.9)$ & \\
\hline Former & $262(45.1)$ & $153(26.3)$ & $109(18.8)$ & \\
\hline Current & $46(7.9)$ & $24(4.1)$ & $22(3.8)$ & 0.10 \\
\hline Hypertension (\%) & $288(49.6)$ & $162(27.9)$ & $126(21.7)$ & 0.19 \\
\hline Diabetes $(\%)$ & $61(10.5)$ & $42(7.2)$ & $19(3.3)$ & 0.01 \\
\hline High cholesterol (\%) & $245(42.2)$ & $135(23.2)$ & $110(18.9)$ & 0.52 \\
\hline Cardiovascular disease $(\%)$ & $161(27.7)$ & $105(18.1)$ & $56(9.6)$ & $>0.001$ \\
\hline Arthritis (\%) & $273(47.0)$ & $125(21.5)$ & $148(25.5)$ & $>0.001$ \\
\hline Blood circulation problems (\%) & $105(18.1)$ & $46(7.9)$ & $59(10.2)$ & 0.03 \\
\hline No. of subjects & 575 & 308 & 267 & \\
\hline \multicolumn{5}{|l|}{ Fazekas score $(0 / 1 / 2 / 3)$} \\
\hline Periventricular & $17 / 371 / 150 / 37$ & $9 / 199 / 84 / 16$ & $8 / 172 / 66 / 21$ & 0.58 \\
\hline Deep white matter & $85 / 368 / 107 / 15$ & $53 / 201 / 51 / 3$ & $32 / 167 / 56 / 12$ & 0.01 \\
\hline
\end{tabular}

Measures given are mean (SD) for scalar data, and number of participants (percentage) for self-reported and WMH load dichotomous and ordinal data. WMH scores were generated from structural scans acquired as part of the LBC1936 imaging protocol by a trained neuroradiologist (Wardlaw et al. 2011). Differences between male and female subgroups were assessed using an independent samples $t$ test for scalar data, and a $\chi^{2}$ test for dichotomous or ordinal data

MMSE Mini-Mental State Examination (maximum score 30), BMI Body mass index

* 446 (267 male) participants provide data on alcohol consumption

\section{Statistical analysis}

Voxel-wise statistics were performed on the individual white matter skeletons using FSL's 'Randomise' tool with 10,000 permutations; threshold-free cluster enhancement (TFCE) correction was applied to the resulting $p$ value volumes to correct for multiple hypothesis testing. A corrected $p$ value of less than 0.05 was considered statistically significant. All results are displayed as regions of significant voxels overlaid on the mean white matter skeleton, with the red/yellow colour scale given by thresholds set between 0.95 and 0.999 in FSLView.

A total of twenty-four linear regression models, of eight types, were fitted to the data to investigate relationships between FA in the white matter skeletons, childhood intelligence (age 11 IQ) and current measures of processing speed, controlling for age and sex. Equation (1) represents the first and simplest model

$\mathrm{FA}=\beta_{0}+\beta_{1} \times$ age $+\beta_{2} \times \operatorname{sex}+\varepsilon$, where $\beta_{0}$ is the intercept, $\beta_{1}$ and $\beta_{2}$ represent the coefficients of age and sex, respectively, and $\varepsilon$ is the error term. Equations (2)-(8) describe the remaining model types, each of them predicting FA from age, sex and other variables of interest indexed by $\beta_{3}$ and $\beta_{4}$

$\mathrm{FA}=\beta_{0}+\beta_{1} \times$ age $+\beta_{2} \times \operatorname{sex}+\beta_{3} \times$ age $11 \mathrm{IQ}+\varepsilon$

$\mathrm{FA}=\beta_{0}+\beta_{1} \times$ age $+\beta_{2} \times \operatorname{sex}+\beta_{3} \times g_{\text {speed }}+\varepsilon$

$\mathrm{FA}=\beta_{0}+\beta_{1} \times$ age $+\beta_{2} \times \operatorname{sex}+\beta_{3}$ $\times$ individual test scores $+\varepsilon$

$\mathrm{FA}=\beta_{0}+\beta_{1} \times$ age $+\beta_{2} \times \operatorname{sex}+\beta_{3}$ $\times$ residual test scores $+\varepsilon$

$\mathrm{FA}=\beta_{0}+\beta_{1} \times$ age $+\beta_{2} \times \operatorname{sex}+\beta_{3} \times g_{\text {speed }}+\beta_{4}$ $\times$ age $11 \mathrm{IQ}+\varepsilon$

$\mathrm{FA}=\beta_{0}+\beta_{1} \times$ age $+\beta_{2} \times \operatorname{sex}+\beta_{3}$ $\times$ individual test scores $+\beta_{4} \times$ age 11 IQ $+\varepsilon$ 
Table 2 Participant mean (SD) values for age 11 IQ, and the information processing speed tests and their residuals

\begin{tabular}{|c|c|c|c|c|}
\hline & \multirow[t]{2}{*}{ Total sample } & \multicolumn{2}{|l|}{ Sex } & \multirow[t]{2}{*}{$p$ value } \\
\hline & & Male & Female & \\
\hline No. of subjects & 581 & 311 & 270 & \\
\hline Age 11 IQ & $101.12(15.21)$ & $99.71(16.43)$ & $102.75(13.51)$ & $>0.02$ \\
\hline Symbol Search & $24.91(6.14)$ & $24.75(6.28)$ & $25.09(5.97)$ & 0.50 \\
\hline Digit-Symbol Substitution & $57.04(12.21)$ & $55.01(12.3)$ & $59.38(11.70)$ & $>0.001$ \\
\hline Simple Reaction Time (s) & $0.27(0.05)$ & $0.27(0.05)$ & $0.27(0.05)$ & 0.53 \\
\hline Four-Choice Reaction Time (s) & $0.64(0.09)$ & $0.64(0.09)$ & $0.64(0.08)$ & 0.96 \\
\hline Inspection Time & $111.75(11.15)$ & $112.87(11.39)$ & $110.47(10.74)$ & 0.009 \\
\hline Residual SS & $0.04(3.67)$ & $0.13(3.71)$ & $-0.06(3.62)$ & 0.53 \\
\hline Residual DS & $0.16(6.38)$ & $-1.34(5.97)$ & $1.89(6.41)$ & $>0.001$ \\
\hline Residual SRT (s) & $6.88 \times 10^{-5}(0.04)$ & $-2.39 \times 10^{-3}(0.04)$ & $2.90 \times 10^{-3}(0.05)$ & 0.15 \\
\hline Residual CRT (s) & $4.30 \times 10^{-4}(0.05)$ & $-2.89 \times 10^{-3}(0.05)$ & $4.26 \times 10^{-3}(0.05)$ & 0.11 \\
\hline Residual IT & $0.11(9.25)$ & $1.56(9.14)$ & $-1.57(9.10)$ & $>0.001$ \\
\hline
\end{tabular}

'Residual' refers to variables that have been residualised for $g_{\text {speed }}$

Differences between male and female subgroups were assessed using an independent samples $t$ test

SS Symbol Search (units: number of rows inspected within the allocated time), DS Digit-Symbol Substitution (units: number of symbols decoded within the allocated time), SRT Simple Reaction Time (units: mean reaction time of correct responses in seconds), CRT Four-Choice Reaction Time (units: mean reaction time of correct responses in seconds), IT Inspection Time (units: number of correct responses)

Table 3 Pearson's product-moment $(r)$ correlations between the individual test scores and the general factor of information processing speed $\left(g_{\text {speed }}\right)$

\begin{tabular}{llllll}
\hline & SS & DS & CRT & SRT & IT \\
\hline SS & - & & & & \\
DS & 0.6252 & - & & & \\
CRT & -0.5011 & -0.5565 & - & & \\
SRT & -0.256 & -0.2489 & 0.4855 & - & \\
IT & 0.371 & 0.3976 & -0.3842 & -0.2641 & - \\
$g_{\text {speed }}^{*}$ & 0.6843 & 0.8823 & -0.5885 & -0.3119 & 0.7742 \\
\hline
\end{tabular}

SS Symbol Search (units: number of rows inspected within the allocated time), $D S$ Digit-Symbol Substitution (units: number of symbols decoded within the allocated time), SRT Simple Reaction Time (units: mean reaction time of correct responses in seconds), CRT FourChoice Reaction Time (units: mean reaction time of correct responses in seconds), IT Inspection Time (units: number of correct responses)

* These correlations are between each test and the component of which the test is a part. They thus give an indication of the loading of each test on the component

$$
\begin{aligned}
\mathrm{FA}= & \beta_{0}+\beta_{1} \times \text { age }+\beta_{2} \times \operatorname{sex}+\beta_{3} \\
& \times \text { residual test scores }+\beta_{4} \times \text { age } 11 \mathrm{IQ}+\varepsilon .
\end{aligned}
$$

The individual processing speed tests (model types in Eqs. 4 and 7) were Digit-Symbol Substitution, Symbol Search, Simple Reaction Time, Four-Choice Reaction Time and Inspection Time described above. Since these individual processing speed tests were highly correlated, each was considered in a separate regression model. Similarly, residual test scores from each test (model types in Eqs. 5 and 8) were investigated separately. In all analyses, age in days at the time of scanning was used, and no model was tested that is not presented above.

\section{Results}

Amongst the 730 participants who underwent MRI, 581 subjects (311 males) fulfilled the inclusion criteria of providing complete DT-MRI, childhood intelligence and processing speed data. The mean age at time of scanning was $72.7 \pm 0.7$ (range 71.0-74.2) years. Table 1 shows participant health characteristics for the whole sample, and male and female subgroups. In general, the male and female subgroups had fairly similar health characteristics, although males had significantly lower Mini-Mental State Examination scores, and significantly higher alcohol consumption, and self-reported incidence of diabetes and cardiovascular disease; conversely females had significantly higher incidence of arthritis and blood circulation problems. The majority of subjects had WMH loads rated on the two lowest Fazekas scores for both periventricular $(67.5 \%)$ and deep $(78.8 \%)$ white matter; females had a significantly higher Fazekas score in deep white matter compared with males. 
Fig. 1 a Associations between white matter FA, age and sex (Eq. 1), b sex differences identified in the same model. Note that this pattern of significant voxels is similar to that observed for sex in all other models. In both figures, regions of significant voxels (red/ yellow) are overlaid on the mean white matter skeleton (green). The effects were thresholded using TFCE at $p<0.05$, with yellow indicating smaller $p$ values (a)

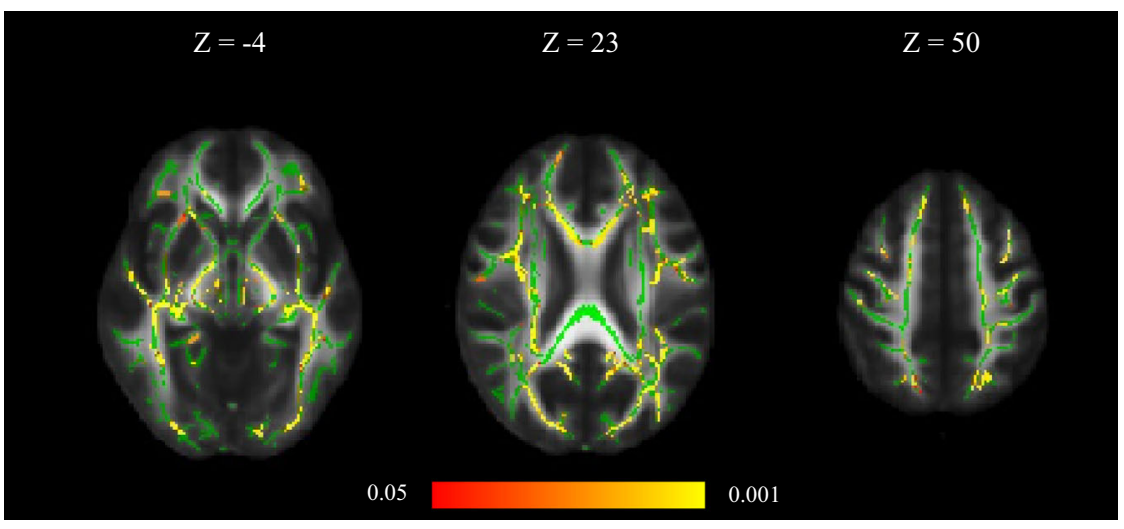

(b)

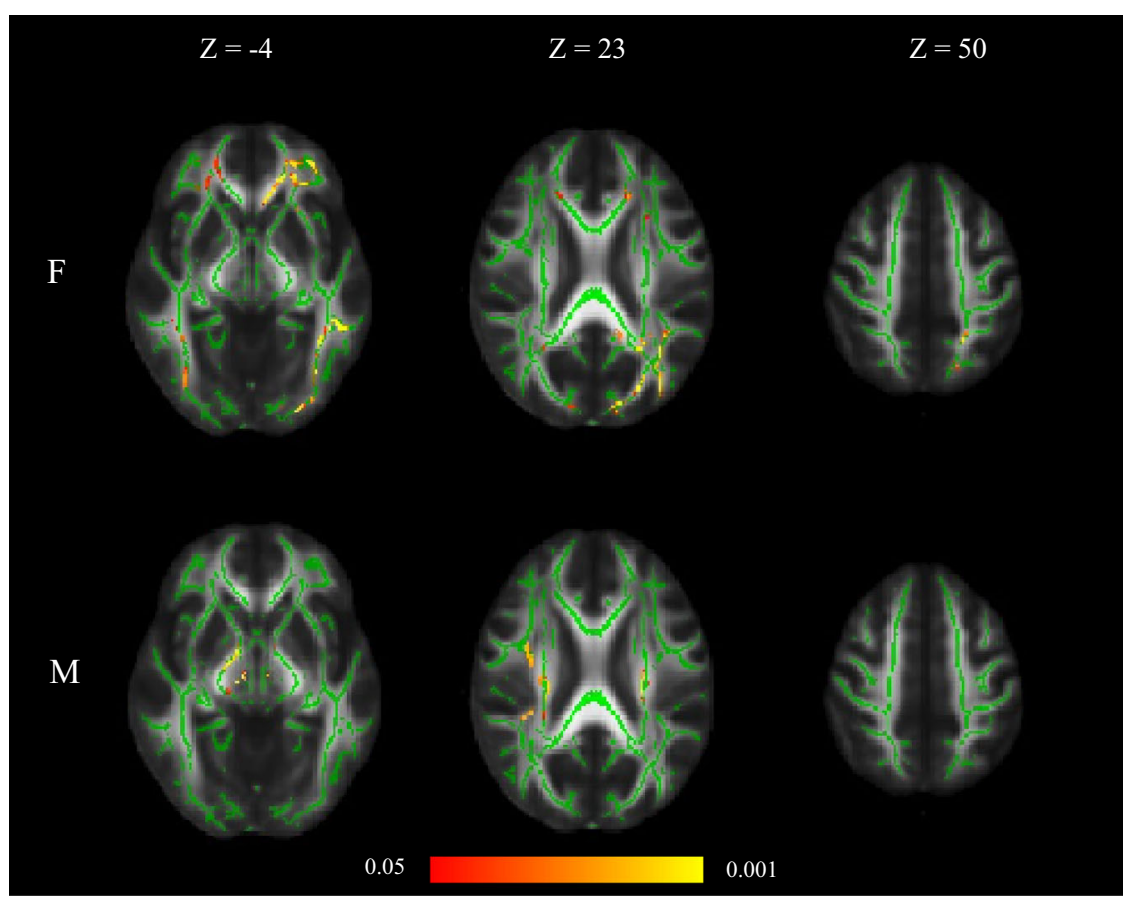

Mean and SD values for age $11 \mathrm{IQ}$, and the processing speed tests and their residuals are shown in Table 2. Males and females performed similarly on these tests, although females had significantly higher age 11 IQ and provided a significantly larger number of correctly decoded symbols in the Digit-Symbol Substitution test than males, while males produced a significantly greater number of correct responses in the Inspection Time task than females. As indicated in Table 3, Pearson's productmoment correlations between the individual processing speed tests were moderate to large suggesting that a common factor could be extracted. As described above, PCA was then run on these data which produced a strong common factor, $g_{\text {speed }}$, which explained $65 \%$ of the total test variance; all cognitive tests had a large correlation with $g_{\text {speed }}$, except Simple Reaction Time which had a moderate correlation.

\section{Age and sex}

The first model (Eq. 1) investigated age and sex as the only predictors of white matter FA. Age, even in this narrow age sample, showed significant negative skeleton-wide associations with white matter FA (Fig. 1a). Sex effects were much less pronounced. Females had significantly higher FA than men, mainly in isolated parts of the genu and splenium of corpus callosum, whereas males had higher FA in small midbrain segments of the superior corona radiata (Fig. 1b).

\section{Childhood intelligence}

The second model (Eq. 2) investigated relationships between childhood intelligence and older age white matter FA. In this model with age and sex, childhood intelligence was not significantly correlated with white matter FA in any voxel. 
Fig. 2 a Associations between white matter FA, $g_{\text {speed }}$, age and sex (Eq. 3), b associations between white matter FA, $g_{\text {speed }}$, childhood intelligence, age and sex (Eq. 6). Regions of significant voxels (red/yellow) are overlaid on the mean white matter skeleton (green). The effects were thresholded using TFCE at $p<0.05$, with yellow indicating smaller $p$ values

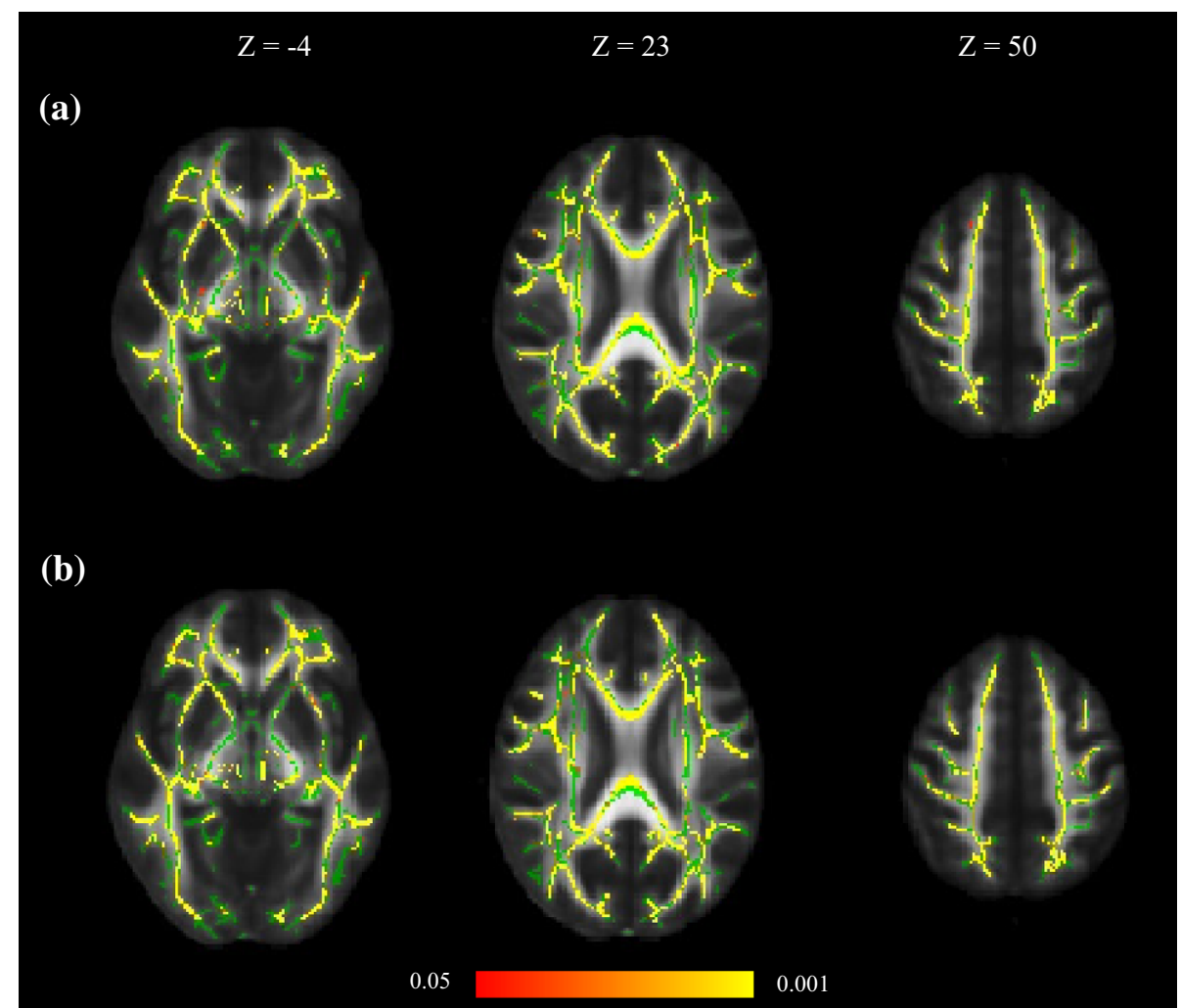

\section{General factor of information processing speed}

Results from the third model (Eq. 3) showed that the general information processing speed factor, $g_{\text {speed }}$, had significant positive skeleton-wide associations with white matter FA after controlling for age and sex (Fig. 2 upper panels). This association remained (and was not significantly weakened) when childhood intelligence was added as a further covariate (Eq. 6; Fig. 2 lower panels). With $g_{\text {speed }}$ included in the model, age was no longer significantly associated with white matter FA; the association with sex remained.

\section{Individual tests of information processing speed}

All five individual processing speed tests were significantly associated with white matter FA across the white matter skeleton after controlling for age and sex (Eq. 4; Fig. 3). Higher scores on Digit-Symbol Substitution, Symbol Search and Inspection Time were positively correlated with white matter FA, whereas faster (lower) Simple and FourChoice Reaction Time were negatively correlated. The directions of association were therefore all consistent with higher FA being associated with faster processing speed. Age was also significantly negatively associated with white matter FA across the white matter skeleton in all models, apart from with Four-Choice Reaction Time, where the age effect was attenuated to non-significance. Sex differences were evident in all models apart from those including Simple and Four-Choice Reaction Time, where sex did not significantly contribute to explaining the variance in FA. When childhood intelligence was added as a covariate (Eq. 7), it did not significantly contribute to explaining the variance in FA in any of the models, but did slightly decrease the significance with which Symbol Search and Inspection Time related to FA. Age remained a significant predictor of white matter FA at age 73 for all five processing speed tests.

\section{Residual analysis of individual cognitive tests contributions}

Finally, we analysed the residuals of each processing speed test after removing the shared variance provided by $g_{\text {speed }}$ and additionally controlling for age and sex (Eq. 5). This model asks whether the specific cognitive capabilities associated with each processing speed task, stripped of that task's association with $g_{\text {speed, }}$, is associated with FA. Figure 4a shows that residual Four-Choice Reaction Time was significantly negatively associated with FA predominantly in the genu of corpus callosum. Residual Digit-Symbol Substitution was significantly positively associated with FA more generally in frontal white matter (Fig. 4b). Residual Symbol Search, Simple Reaction Time and Inspection Time did not have 


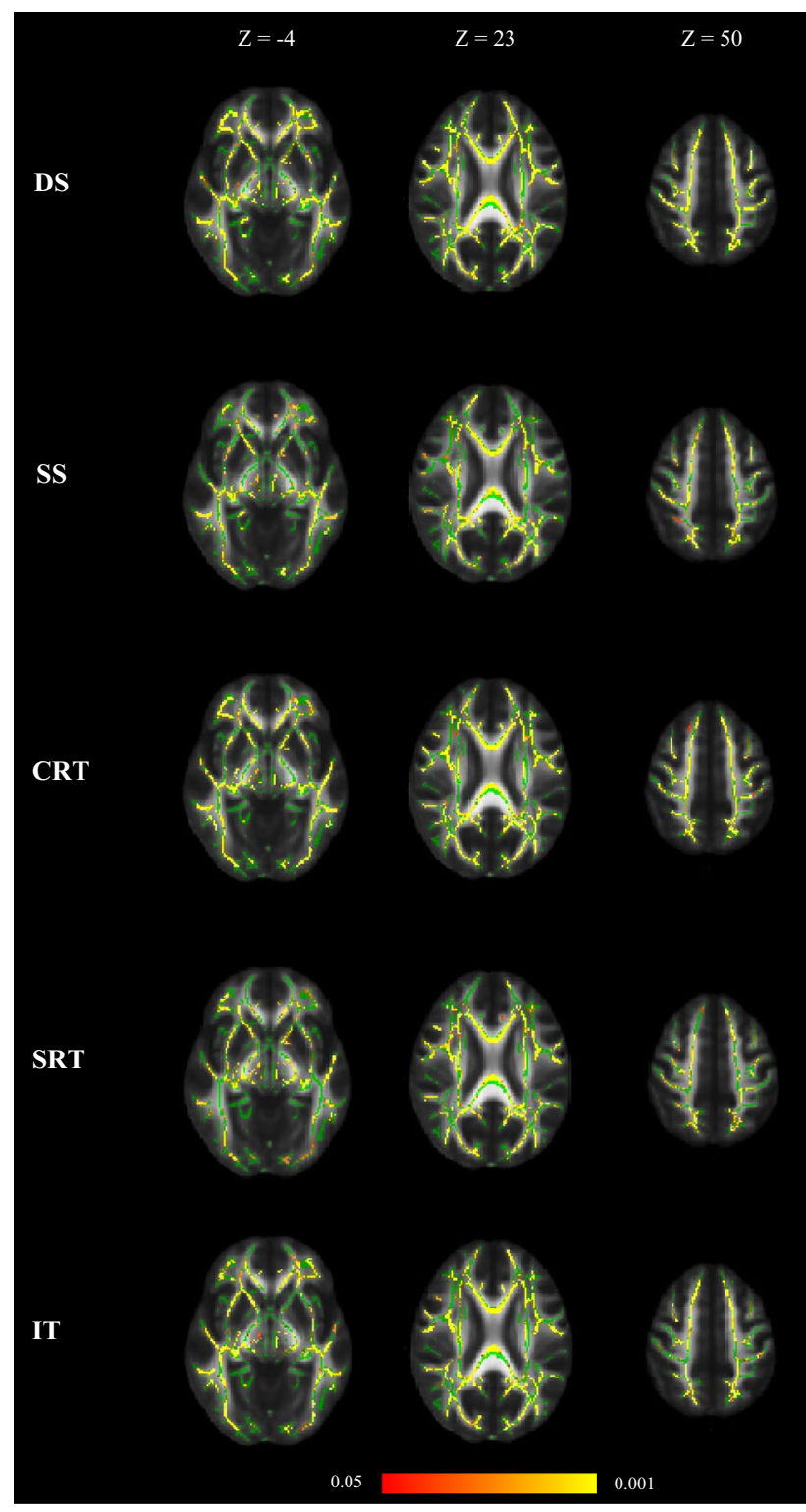

Fig. 3 Associations between white matter FA, individual information processing speed tests, age and sex (Eq. 4); DS Digit-Symbol Substitution, SS Symbol Search, CRT Four-Choice Reaction Time, $S R T$ Simple Reaction Time and IT Inspection Time. Regions of significant voxels (red/yellow) are overlaid on the mean white matter skeleton (green). The effects were thresholded using TFCE at $p<0.05$, with yellow indicating smaller $p$ values

significant associations with $\mathrm{FA}$; that is, their associations with FA were accounted for by the variance these tests shared with other processing speed tests. When childhood intelligence was added as a covariate (Eq. 8), it had a significant positive association with FA principally in the splenium and posterior body of corpus callosum in all residual models apart from Digit-Symbol Substitution where there was no association (Fig. 5). Age remained a significant predictor of white matter FA in all models.

\section{Discussion}

The present study used voxel-based methods to extend previous analyses that have employed quantitative tractography to investigate links between cognitive ability and white matter structure in the LBC1936 (Penke et al. 2010, 2012). The results showed that, in older age, there were skeleton-wide associations between white matter FA and measures of processing speed which supports the hypothesis that the latter is not localised to specific tracts (Haász et al. 2013; Penke et al. 2010, 2012). This was the case regardless of whether individual tests or $g_{\text {speed }}$ was used to index processing speed. There were, however, some specific areas of the brain that were associated with variance in some tasks that were not part of general processing speed. Finally, intelligence assessed at age 11 years was not associated with white matter FA in most models suggesting that older age associations between white matter structure and processing speed are generally not accounted for by cognitive ability measured in youth. Our findings highlight the crucial role white matter plays in providing a neuroanatomical structure for individual differences in the information transfer underpinning fundamental aspects of human intelligence. They also provide support for viewing the brain as a highly connected distributed network with the slower processing speed found in older age being part of a process of cortico-cortical disconnection (Bennett and Madden 2014; Felleman and Van Essen 1991; Sporns 2013).

Beyond $g_{\text {speed }}$, we investigated whether the individual processing speed tests had specific associations with white matter FA. This was achieved by removing the shared variance with all the other tests from each individual test by residualising for $g_{\text {speed }}$. The residuals of Symbol Search, Simple Reaction Time and Inspection Time were no longer associated with white matter FA, indicating that variance specific to these tests was not related to white matter structure beyond their shared variance with $g_{\text {speed }}$. However, residual Four-Choice Reaction Time was significantly associated with FA in the genu of corpus callosum, and residual DigitSymbol Substitution was associated with FA in frontal white matter. This may be due to these tasks' overlap with cognitive abilities other than information processing speed. For example, there is evidence that the Digit-Symbol Substitution task also involves use of memory which is associated with frontal fibres (Charlton et al. 2010; Piccinin and Rabbitt 1999). Similarly, faster responses on Four-Choice Reaction Time require inter-hemispheric coordination and selection of manual responses, which is associated with the corpus callosum (Doron and Gazzaniga 2008).

Childhood intelligence was not significantly associated with older age white matter FA in the model with age and 
Fig. 4 a Associations between white matter FA, residual FourChoice Reaction Time, age and sex (Eq. 5), b associations between white matter FA, residual Digit-Symbol

Substitution, age and sex (Eq. 5). Regions of significant voxels (red/yellow) are overlaid on the mean white matter skeleton (green). The effects were thresholded using TFCE at $p<0.05$, with yellow indicating smaller $p$ values (a)

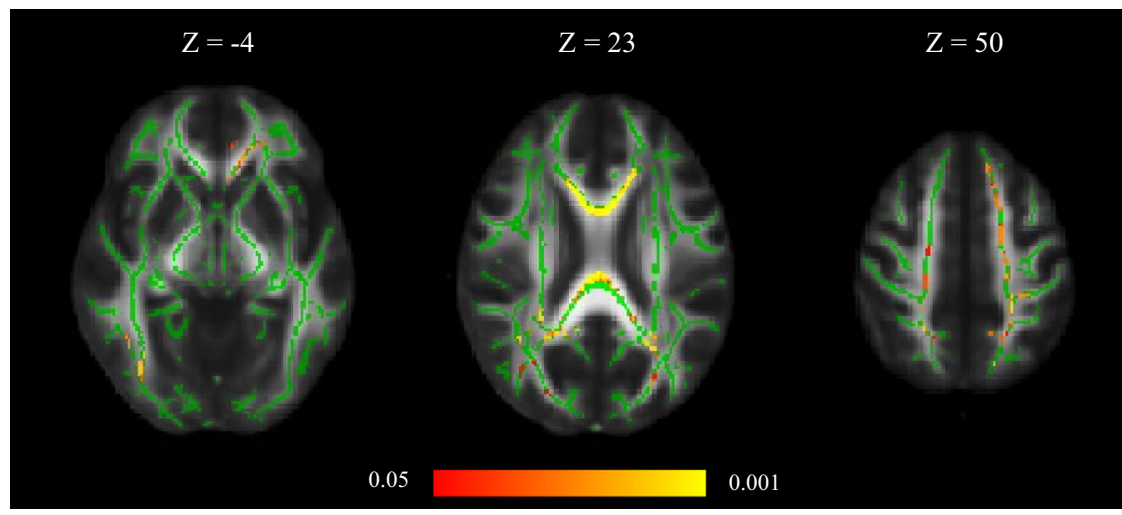

(b)

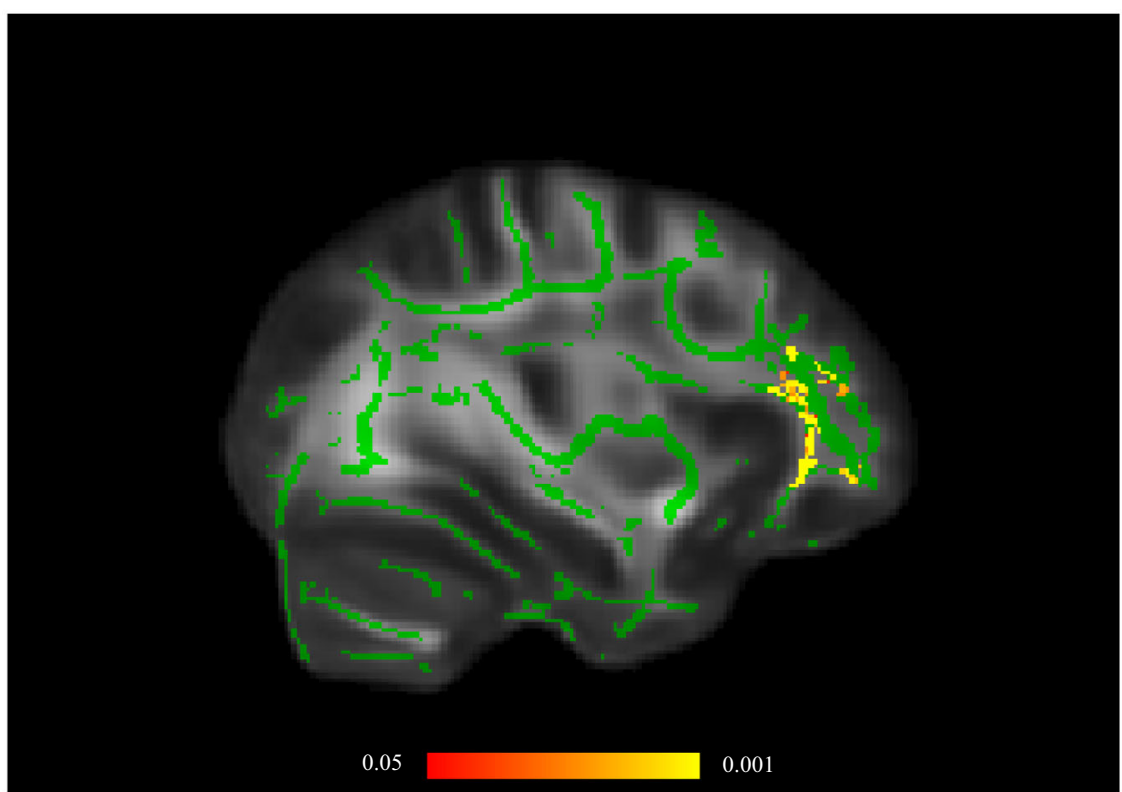

sex as the only covariates. Similarly, there were no significant associations between childhood intelligence and white matter FA when $g_{\text {speed }}$, or any of its individual processing speed tests, were also included in the model. However, when the same analysis was repeated with residual processing speed scores, childhood intelligence became significantly associated with white matter FA in the splenium and posterior body of corpus callosum for all residual scores except Digit-Symbol Substitution. These data therefore suggest that although early life intelligence does not strongly predict later life global white matter FA, it does predict the specific relationship between splenium FA and better scores on the $g_{\text {speed-independent components }}$ of these individual cognitive tests. A possible explanation for this finding is that the residual test scores reflect other orthogonal cognitive domains to processing speed, such as memory, which are known to relate to measures of childhood cognitive ability (Deary 2014). These results should be considered in the context of other analyses performed in the LBC1936, for example regarding WMH burden and brain volume, which show that early life cognitive function has a significant effect on white matter structure in older age. Specifically, Valdés Hernández et al. (2013) found in approximately 600 LBC1936 participants that childhood intelligence was negatively correlated with $\mathrm{WMH}$ volume $(r=-0.08)$ suggesting that lower cognitive ability in youth leads to increasing white matter damage with age, while Royle et al. (2013) reported that childhood intelligence was a predictor of estimated prior $(r=0.12)$ and measured current brain volume $(r=0.11)$ in older age. ${ }^{1}$ However, Penke et al. (2012) found that white matter FA measured in 12 major tracts using quantitative tractography was more associated with intelligence in later rather than early life, as reported here. It is therefore possible that DT-

\footnotetext{
1 Although not involving brain white matter structure, another LBC1936 analysis also emphasised the importance of having cognitive test data from early life. Specifically, Karama et al. (2014) found that widespread cross-sectional associations at age 73 years between brain cortical thickness and cognitive ability were wholly confounded by cognitive ability tested at age 11 .
} 
Fig. 5 Associations between white matter FA, childhood intelligence, age, sex and residual (left to right) FourChoice Reaction Time (CRT), Simple Reaction Time (SRT), Symbol Search (SS) and Inspection Time (IT) (Eq. 8). Regions of significant voxels (red/yellow) are overlaid on the mean white matter skeleton (green). The effects were thresholded using TFCE at $p<0.05$, with yellow indicating smaller $p$ values

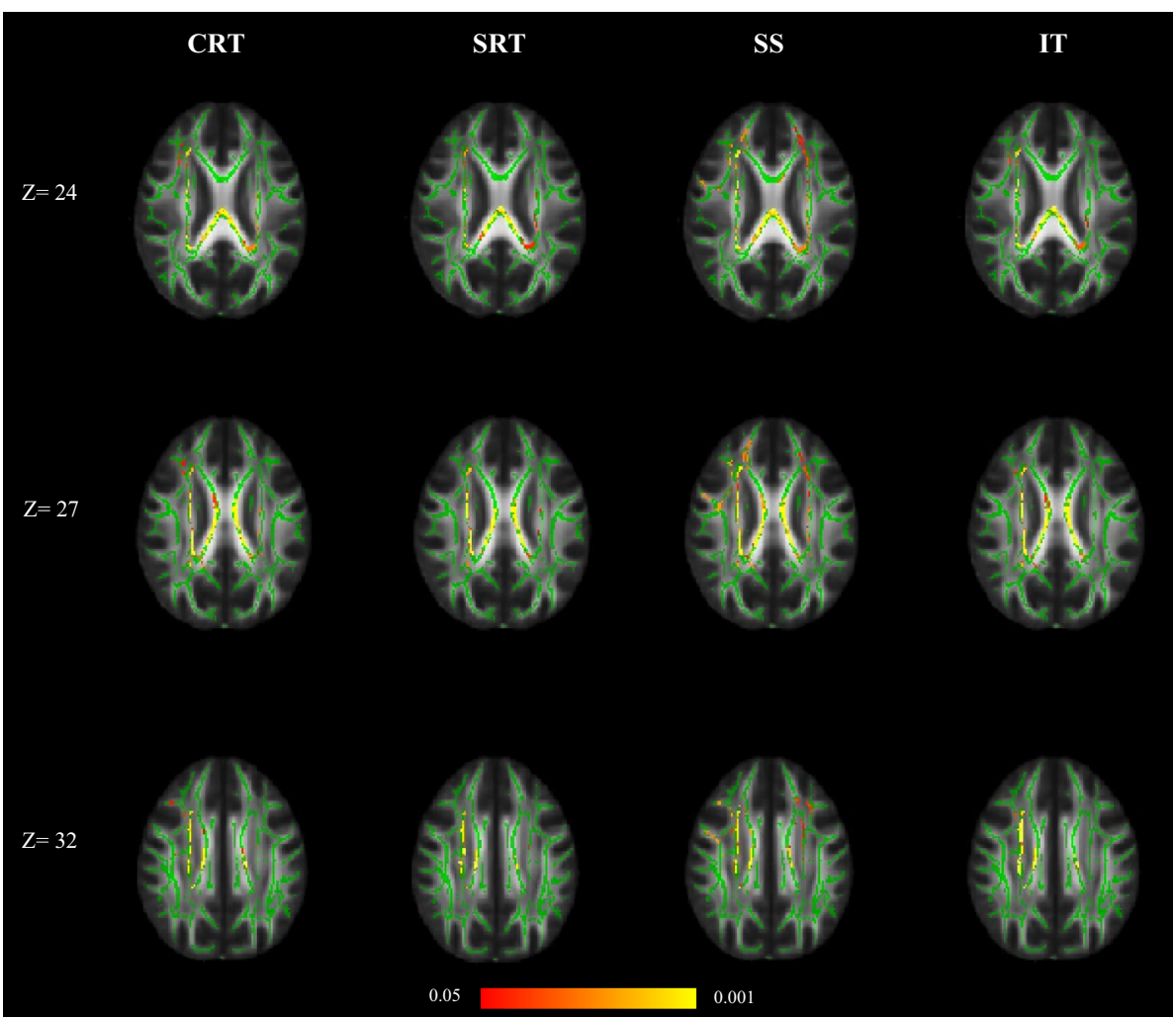

MRI biomarkers, even measured skeleton-wide in voxelbased analyses, reflect the effects of cognitive ageing more than they reflect lifelong-stable differences in intelligence. Clearly, the availability of measures of childhood cognitive ability is an uncommon feature of the current study which makes generalising these findings more difficult; it would be tempting to conclude that early life cognition need not be a key feature of DT-MRI studies of normal ageing. Further work, in both cross-sectional and longitudinal analyses, in cohorts with such early life cognitive data, for example the Aberdeen Birth Cohorts (http://www.abdn.ac. uk/birth-cohorts), is required to clarify this finding.

Our results showed some subtle sex differences: females had higher white matter FA in isolated parts of the genu and splenium of corpus callosum, whereas males had higher white matter FA in small mid-brain segments of the superior corona radiata. This may reflect differential brain ageing according to sex, although males and females had fairly similar healthy characteristics in this cohort. These sex differences in white matter structure will require replication in other large, later life cohorts.

This study has several strengths compared to previous studies of information processing speed and white matter structure in older age. The LBC1936 is a large cohort with a narrow age range and generally healthy participants. Subjects took five processing speed tasks drawn from different traditions, namely psychometric, experimental psychological and psychophysical tests. Together, they created a reliable measure of $g_{\text {speed. }}$. Furthermore, the availability of childhood intelligence data from age 11 allows prior cognitive ability to be controlled for and thus permits changes that are specifically due to ageing to be identified more efficiently. This is an important issue since we have shown previously that all of the processing speed tests are strongly associated with general cognitive ability from youth, about 60 years earlier (Deary et al. 2010). Analysis of the DT-MRI data using TBSS allows relations between processing speed and white matter structure to be investigated voxel-by-voxel across the brain, rather than in a few major tracts, in an automated and user-independent fashion. However, this method only allows comparison of individual differences in white matter FA across those areas which show good spatial concordance with the white matter skeleton. Thus, local gyral white matter and other areas, which show a high degree of morphological variability, were not included in these analyses.

The narrow age range of this sample can, conversely, also be viewed as a limitation because our findings cannot be generalised to younger populations and are only representative of older subjects. Similarly, because the LBC1936 subjects are generally healthy, our finding cannot be directly applied to ageing populations with decreased processing speed due to neurodegenerative diseases, such as dementia. In order to keep the number of models 
manageable, we did not investigate associations between processing speed test scores and other DT-MRI biomarkers, such as mean, radial and axial diffusivity; these variables may be more prone to partial volume CSFcontamination errors than FA (Metzler-Baddeley et al. 2012). Finally, we did not take into account WMH; how these features might affect the results reported here is currently being investigated in the subgroup of LBC1936 participants who have significant lesion burden.

In conclusion, we found using voxel-based analyses that $g_{\text {speed }}$, as well as five varied tests of information processing speed, had significant skeleton-wide associations with white matter FA. These results indicate that quicker and more efficient information processing requires global connectivity in older age. Conversely, they can also be interpreted to imply that there is an age-related global reduction in connectivity that affects all networks, including those associated with the administered processing speed tests. Childhood intelligence was not associated with white matter FA, except for several residual $\left(g_{\text {speed }}\right.$-independent) test score models, suggesting that associations between white matter structure and information processing speed measured in older age are not generally accounted for by cognitive ability measured in youth.

Acknowledgments The work was undertaken as part of the Cross Council and University of Edinburgh Centre for Cognitive Ageing and Cognitive Epidemiology (CCACE; http://www.ccace.ed.ac.uk). This work was supported by a Research into Ageing programme grant (to I.J.D. and J.M.S.) and the Age UK-funded Disconnected Mind project (http://www.disconnectedmind.ed.ac.uk; to I.J.D., J.M.S. and J.M.W.), with additional funding from the UK Medical Research Council (MRC; to I.J.D., J.M.S., J.M.W., M.E.B., S.J.R. and S.R.C.). The University of Edinburgh School of Informatics Doctoral Training Centre in Neuroinformatics and Computational Neuroscience (http:// www.anc.ac.uk/dtc), of which K.A.K. is a member, was supported in part by grants EP/F500385/1 and BB/F529254/1 from the UK Engineering and Physical Sciences Research Council (EPSRC), UK Biotechnology and Biological Sciences Research Council (BBSRC), and MRC. J.M.W. was supported by the Scottish Funding Council through the SINAPSE Collaboration (http://www.sinapse.ac.uk). CCACE (MRC MR/K026992/1) was funded by the BBSRC and MRC. The image acquisition and analysis was performed at the Brain Research Imaging Centre, University of Edinburgh (http://www.bric. ed.ac.uk).

\section{Compliance with ethical standards}

Conflict of interest The authors declare that they have no actual or potential conflicts of interest with this work.

Open Access This article is distributed under the terms of the Creative Commons Attribution 4.0 International License (http://crea tivecommons.org/licenses/by/4.0/), which permits unrestricted use, distribution, and reproduction in any medium, provided you give appropriate credit to the original author(s) and the source, provide a link to the Creative Commons license, and indicate if changes were made.

\section{References}

Bartzokis G, Beckson M, Lu PH, Nuechterlein KH, Edwards N, Mintz J (2001) Age-related changes in frontal and temporal lobe volumes in men: a magnetic resonance imaging study. Arch Gen Psychiatry 58:461-465

Bartzokis G, Tishler TA, Lu PH, Villablanca P, Altshuler LL, Carter M, Huang D, Edwards N, Mintz J (2007) Brain ferritin iron may influence age- and gender-related risks of neurodegeneration. Neurobiol Aging 28:414-423

Bennett IJ, Madden DJ (2014) Disconnected aging: cerebral white matter integrity and age-related differences in cognition. Neuroscience 276:187-205

Booth T, Bastin ME, Penke L, Maniega SM, Murray C, Royle NA, Gow AJ, Corley J, Henderson RD, Hernández MC, Starr JM, Wardlaw JM, Deary IJ (2013) Brain white matter tract integrity and cognitive abilities in community-dwelling older people: the Lothian Birth Cohort, 1936. Neuropsychology 27:595-607

Charlton RA, Barrick TR, Lawes IN, Markus HS, Morris RG (2010) White matter pathways associated with working memory in normal aging. Cortex 46:474-489

Cox BD, Huppert FA, Whichelow MJ (1993) The Health and Lifestyle Survey: seven years on. Dartmouth, Aldershot

Deary IJ (2000) Looking down on human intelligence: from psychometrics to the brain. Oxford University Press, Oxford

Deary IJ (2014) The stability of intelligence from childhood to old age. Curr Dir Psych Sci 23:239-245

Deary IJ, Der G, Ford G (2001) Reaction times and intelligence differences: a population-based cohort study. Intelligence 29:389-399

Deary IJ, Simonotto E, Meyer M, Marshall A, Marshall I, Goddard N, Wardlaw JM (2004) The functional anatomy of inspection time: an event-related fMRI study. NeuroImage 22:1466-1479

Deary IJ, Gow AJ, Taylor MD, Corley J, Brett C, Wilson V, Campbell H, Whalley LJ, Visscher PM, Porteous DJ, Starr JM (2007) The Lothian Birth Cohort 1936: a study to examine influences on cognitive ageing from age 11 to age 70 and beyond. BMC Geriatr 7:28

Deary IJ, Corley J, Gow AJ, Harris SE (2009) Age-associated cognitive decline. Br Med Bull 92:135-152

Deary IJ, Johnson W, Starr JM (2010) Are processing speed tasks biomarkers of cognitive aging? Psychol Aging 25:219-228

Deary IJ, Gow AJ, Pattie A, Starr JM (2012) Cohort profile: the Lothian Birth Cohorts of 1921 and 1936. Int J Epidemiol 41:1576-1584

Doron KW, Gazzaniga MS (2008) Neuroimaging techniques offer new perspective on callosal transfer and interhemispheric communication. Cortex 44:1023-1029

Doubal FN, MacLullich AM, Ferguson KJ, Dennis MS, Wardlaw JM (2010) Enlarged perivascular spaces on MRI are a feature of cerebral small vessel disease. Stroke 41:450-454

Fazekas F, Chawluk JB, Alavi A, Hurtig HI, Zimmerman RA (1987) MR signal abnormalities at $1.5 \mathrm{~T}$ in Alzheimer's dementia and normal aging. AJR Am J Roentgenol 149:351-356

Felleman DJ, Van Essen DC (1991) Distributed hierarchical processing in the primate cerebral cortex. Cereb Cortex 1:1-47

Gunning-Dixon FM, Brickman AM, Cheng JC, Alexopoulos GS (2009) Aging of cerebral white matter: a review of MRI findings. Int J Geriatr Psychiatry 24:109-117

Haász J, Westlye ET, Fjær S, Espeseth T, Lundervold A, Lundervold AJ (2013) General fluid-type intelligence is related to indices of white matter structure in middle-aged and old adults. NeuroImage 83:372-383 
Hofer SM, Sliwinski MJ (2001) Understanding ageing. An evaluation of research designs for assessing the interdependence of ageingrelated changes. Gerontology 47:341-352

Jenkinson M, Smith S (2001) A global optimisation method for robust affine registration of brain images. Med Image Anal 5:143-156

Jensen AR (1987) Process differences and individual differences in some cognitive tasks. Intelligence 11:107-136

Jensen AR (2006) Clocking the mind: mental chronometry and individual differences, 1st edn. Elsevier Science, London

Jones DK, Williams SC, Gasston D, Horsfield MA, Simmons A, Howard R (2002) Isotropic resolution diffusion tensor imaging with whole brain acquisition in a clinically acceptable time. Hum Brain Mapp 15:216-230

Jones DK, Knösche TR, Turner R (2013) White matter integrity, fiber count, and other fallacies: the do's and don'ts of diffusion MRI. NeuroImage 73:239-254

Karama S, Bastin ME, Murray C, Royle NA, Penke L, Muñoz Maniega S, Gow AJ, Corley J, Valdés Hernández Mdel C, Lewis JD, Rousseau MÉ, Lepage C, Fonov V, Collins DL, Booth T, Rioux P, Sherif T, Adalat R, Starr JM, Evans AC, Wardlaw JM, Deary IJ (2014) Childhood cognitive ability accounts for associations between cognitive ability and brain cortical thickness in old age. Mol Psychiatry 19:555-559

Kennedy KM, Raz N (2009) Aging white matter and cognition: differential effects of regional variations in diffusion properties on memory, executive functions, and speed. Neuropsychologia 47:916-927

Luciano M, Gow AJ, Harris SE, Hayward C, Allerhand M, Starr JM, Visscher PM, Deary IJ (2009) Cognitive ability at age 11 and 70 years, information processing speed, and APOE variation: the Lothian Birth Cohort 1936 study. Psychol Aging 24:129-138

Madden DJ, Bennett IJ, Burzynska A, Potter GG, Chen NK, Song AW (2012) Diffusion tensor imaging of cerebral white matter integrity in cognitive aging. Biochim Biophys Acta 1822:386-400

Malloy P, Correia S, Stebbins G, Laidlaw DH (2007) Neuroimaging of white matter in aging and dementia. Clin Neuropsychol 21:73-109

Metzler-Baddeley C, O’Sullivan MJ, Bells S, Pasternak O, Jones DK (2012) How and how not to correct for CSF-contamination in diffusion MRI. NeuroImage 59:1394-1403

Penke L, Muñoz Maniega S, Murray C, Gow AJ, Hernández MC, Clayden JD, Starr JM, Wardlaw JM, Bastin ME, Deary IJ (2010) A general factor of brain white matter integrity predicts information processing speed in healthy older people. J Neurosci 30:7569-7574

Penke L, Maniega SM, Bastin ME, Valdés Hernández MC, Murray C, Royle NA, Starr JM, Wardlaw JM, Deary IJ (2012) Brain white matter tract integrity as a neural foundation for general intelligence. Mol Psychiatry 17:1026-1030

Piccinin AM, Rabbitt P (1999) Contribution of cognitive abilities to performance and improvement on a substitution coding task. Psychol Aging 14:539-551

Raz N, Lindenberger U, Rodrigue KM, Kennedy KM, Head D, Williamson A, Dahle C, Gerstorf D, Acker JD (2005) Regional brain changes in aging healthy adults: general trends, individual differences and modifiers. Cereb Cortex 15:1676-1689

Ritchie SJ, Tucker-Drob EM, Deary IJ (2014) A strong link between speed of visual discrimination and cognitive ageing. Curr Biol 24:R681-R683
Royle NA, Booth T, Valdés Hernández MC, Penke L, Murray C, Gow AJ, Muñoz Maniega S, Starr JM, Bastin ME, Deary IJ, Wardlaw JM (2013) Estimated maximal and current brain volume predict cognitive ability in old age. Neurobiol Aging 34:2726-2733

Salat DH, Tuch DS, Greve DL, van der Kouwe JW, Hevelone ND, Zaleta K, Rosen BR, Fischl B, Corkin S, Rosas HD, Dale M (2005) Age-related alterations in white matter microstructure measured by diffusion tensor imaging. Neurobiol Aging 26:1215-1227

Salthouse TA (1996) The processing-speed theory of adult age differences in cognition. Psychol Rev 103:403-428

Salthouse TA (2010) Major issues in cognitive aging. Oxford University Press, UK

Scottish Council for Research in Education (1949) The trend of Scottish intelligence: a comparison of the 1947 and 1932 surveys of the intelligence of eleven-year-old pupils. University of London Press, London

Smith SM, Jenkinson M, Johansen-Berg H, Rueckert D, Nichols TE, Mackay CE, Watkins KE, Ciccarelli O, Cader MZ, Matthews PM, Behrens TE (2006) Tract-based spatial statistics: voxelwise analysis of multi-subject diffusion data. NeuroImage $31: 1487-1505$

Sporns O (2013) The human connectome: origins and challenges. NeuroImage 80:53-61

Sullivan EV, Pfefferbaum A (2006) Diffusion tensor imaging and aging. Neurosci Biobehav Rev 30:749-761

Tucker-Drob EM, Briley DA, Starr JM, Deary IJ (2014) Structure and correlates of cognitive aging in a narrow age cohort. Psychol Aging 29:236-249

Valdés Hernández M del C, Booth T, Murray C, Gow AJ, Penke L, Morris Z, Maniega SM, Royle NA, Aribisala BS, Bastin ME, Starr JM, Deary IJ, Wardlaw JM (2013) Brain white matter damage in aging and cognitive ability in youth and older age. Neurobiol Aging 34:2740-2747

Van den Heuvel DM, ten Dam VH, de Craen AJ, Admiraal-Behloul F, Olofsen H, Bollen EL, Jolles J, Murray HM, Blauw GJ, Westendorp RG, van Buchem MA (2006) Increase in periventricular white matter hyperintensities parallels decline in mental processing speed in a non-demented elderly population. J Neurol Neurosurg Psychiatry 77:149-153

Verhaeghen P (2014) The elements of cognitive ageing. Oxford University Press, Oxford

Vernooij MW, Ikram MA, Vrooman HA, Wielopolski PA, Krestin GP, Hofman A, Niessen WJ, Van der Lugt A, Breteler MM (2009) White matter microstructural integrity and cognitive function in a general elderly population. Arch Gen Psychiatry 66:545-553

Wardlaw JM, Bastin ME, Valdes Hernandez MC, Munoz Maniega S, Royle N, Morris Z, Clayden JD, Sandeman E, Eadie E, Murray C, Starr JM, Deary IJ (2011) Brain ageing, cognition in youth and old age, and vascular disease in the Lothian Birth Cohort 1936: rationale, design and methodology of the imaging protocol. Int J Stroke 6:547-559

Wechsler D (1998) WAIS-III ${ }^{\mathrm{UK}}$ administration and scoring manual. Psychological Corporation, London 